\title{
Tele-doc: an open architecture robotic system for telemedicine applications
}

\author{
Anan Osothsilp \\ and Farzad Pourboghrat*
}

Department of Electrical and Computer Engineering, Southern Illinois University, Carbondale, IL 62901 6603, USA

E-mail: anan14@siu.edu

E-mail: pour@siu.edu

${ }^{*}$ Corresponding author

\begin{abstract}
This paper describes a research study for the development and implementation of a prototype remote-controlled robotic assistant to doctors (Tele-Doc) for telemedicine applications. The development considers implementation issues concerning real-time and continuous patient monitoring and diagnosis, as well as tele-control interfacing techniques, mobile robot motion controller, reprogrammable embedded processing system, and internet-based robotic control centre. The paper also describes bio-data measurement techniques, as well as simple system architecture and its implementation.
\end{abstract}

Keywords: mobile robot; telemedicine; human computer interaction; embedded system; web service; XML database system.

Reference to this paper should be made as follows: Osothsilp, A. and Pourboghrat, F. (2008) 'Tele-doc: an open architecture robotic system for telemedicine applications', Int. J. Functional Informatics and Personalised Medicine, Vol. 1, No. 4, pp.390-406.

Biographical notes: Anan Osothsilp is a doctoral student in Electrical and Computer Engineering at Southern Illinois University, Carbondale. His research interests are in the areas of networked embedded control systems and applications.

Farzad Pourboghrat received his PhD Degree in Electrical Engineering from the University of Iowa, Iowa City, in 1984. He is a Professor of Electrical and Computer Engineering at Southern Illinois University at Carbondale (SIUC). His research interests include real-time modelling and control of dynamic systems, robust and adaptive control design and implementation with applications to embedded systems.

\section{Introduction}

Over the past years, interactive tele-visit has become an alternative tool that allows doctors to virtually interact with their patients. This type of telemedicine requires tele-presence through mobile robotic systems which require robust mobility and virtual 
presence (Smith and Skandalakis, 2005; Trahanias et al., 2005). Using current robotic systems (Thacker, 2005), doctors can tele-control such systems for navigation in hospitals and healthcare facilities to visit patients using tele-conference technology. Currently, active areas of research and development for such mobile robotic systems consist of robot's local navigation and visual presence for both outdoor and indoor environments. However, there is no direct physical interaction among doctor, patient, and robot. Moreover, checking and monitoring patient's bio data is currently performed by healthcare professionals using measurement facilities available in the medical environment. In particular, the data are usually obtained at periodic schedules. Although doctors can have access to such information via the hospital's database system, the data is not real-time information and does not accurately render the patient's current condition. For patients staying outside the hospital environment, their bio measurement and data analysis is difficult to obtain. In these situations, doctors would lose track of the patient's condition. For bio data analysis, an additional software module is required. In Moret-Bonillo et al. (1997) and Melek et al. (2005), software packages have been developed to help doctors diagnose patients' conditions using off-line bio data. The data is analysed and compared with expert database information. Similar capabilities could be extended to robotic tele-visit systems under a doctor's supervision. In addition, tele-control techniques together with video presence, robot navigation, and physical interactions, allow doctors to interact with patients via robotic systems.

This research concentrates on the development and implementation of a prototype tele-robot system with an interactive support environment. This will enhance the existing robotic capability to provide better level of interaction, real-time measurement, and bio data organisation for health-care applications. In particular, the proposed prototype requires mobile robot development, an embedded bio measurement system, a multi-task doctor-robot physical interaction, tele-control software, and a patient database system. Figure 1 illustrates the working environment of such a robotic system.

Figure 1 Working environment of the proposed system (see online version for colours)

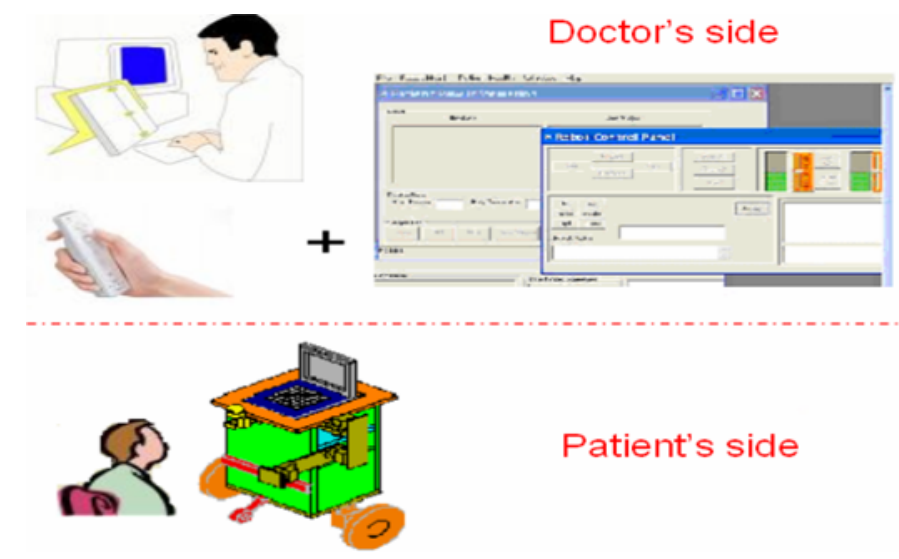

This paper is organised as follows: Section 1 provides a brief introduction to the work; Section 2 describes the robotic system's components; Section 3 focuses on system implementation; Section 4 describes some sensor implementation, and finally, Section 5 concludes the paper and provides some perspectives. 


\section{System components}

The development of the proposed system requires both hardware and software designs. The main hardware components include the robot's mechanical subsystems, sensors, actuators, power modules, wireless communication systems, and embedded processors (Murray et al., 1994). Also, major software components include doctor-side and robot-side routines. A doctor-side routine consists of a doctor's control-desk module for doctor-robot interface and patient data monitoring and a robot interface module for remote navigation and video-conferencing. The robot-side routine consists of health monitoring and patient database service modules, as well as local control codes. Various system interfaces are explained as follows.

\subsection{Doctor-side interface}

The system incorporates a set of embedded hardware and software modules. Virtual control of a robotic platform is achieved using a special wireless joystick (Wii) as the robot's remote controller (Vannoni and Straulino, 2007). Acceleration sensors embedded inside this remote controller detect both acceleration and linear displacement. The relationship between angle and acceleration information for near-static conditions is well documented in Coulston (2005). With Bluetooth wireless capability (Sagiroglu et al., 2006), the joystick can be adopted by the doctor to transform his or her hand's movement to a proper robot movement command. Sensor information, including button pressing and the joystick's linear and rotational motion, is transmitted to the doctor's computer via a Bluetooth device. A hand-motion-pattern software module is then used to compare the generated commands with the doctor's hand movement database. This results in command movements similar to clicking on the move button. For multi-task control, the doctor can swing the joystick and also press particular joystick buttons simultaneously. For instance, pressing button 1 and swinging the remote controller to the left side activates continuous data measurement and moves the pan-tilt camera facing up at the same time. Thus movement pattern can be predefined to fit each doctor's personal preferences. Figure 2 illustrates an example of a pattern database for joystick control.

Figure 2 Pattern database for joystick control (see online version for colours)

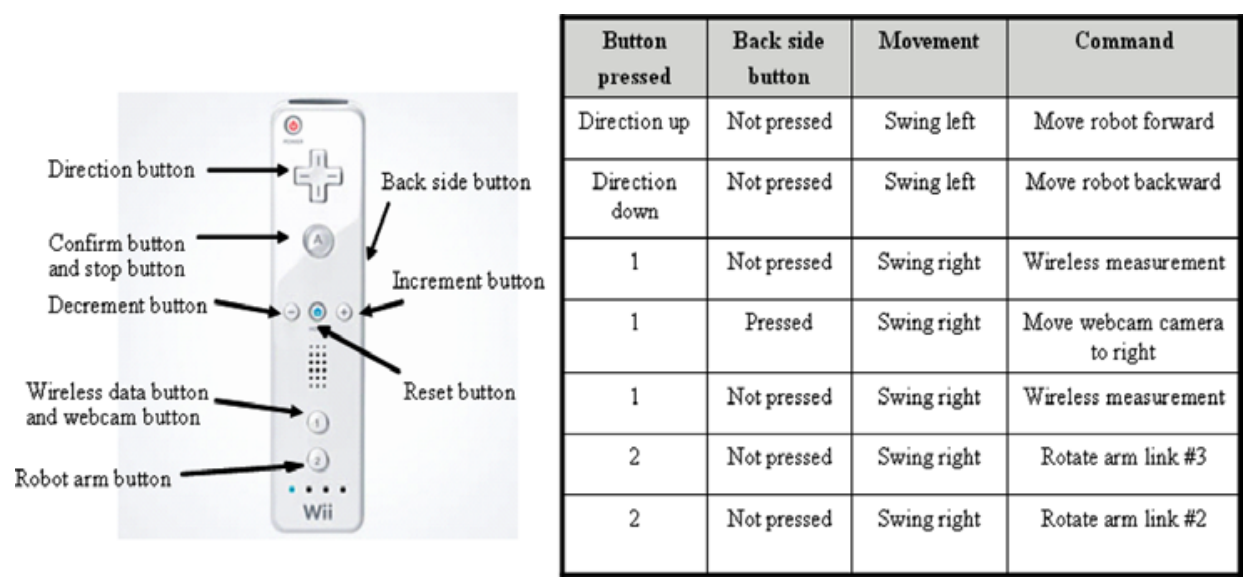


For tele-presence, both image and voice communication modules are implemented and fully integrated into the doctor's control panel module. The patient's data monitoring is also an important addition to the proposed system. Using patient data monitoring software (Bio metering), the doctor can visualise and obtain the patient's bio-data in real-time. A Graphical User Interface (GUI) depicting the doctor's control panel and bio metering display is shown in Figure 3.

Figure 3 Doctor's control panel module and real-time bio metering module (see online version for colours)

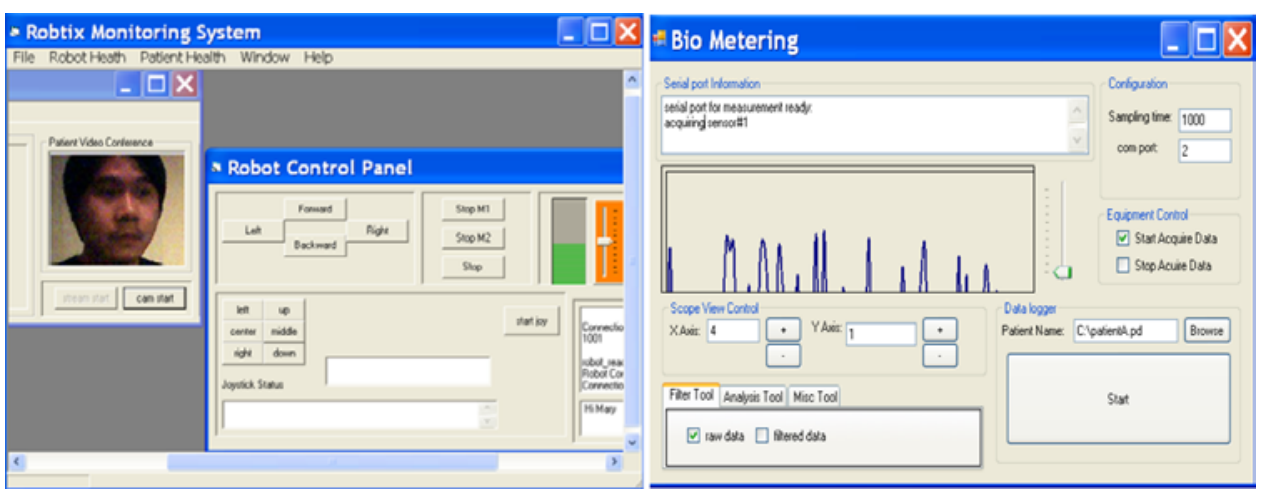

\subsection{Robot side interface}

The robotic system includes the robot structure, laptop and several embedded hardware modules. A Pentium 4 dual core laptop is adopted as the main processing workforce. Reprogrammable embedded systems are integrated into the robotic system to provide multi-functional tasks including actuation and sensing mechanisms. In general, each embedded system unit communicates with the main computer laptop via a USB port. Real-time localisation is realised using a set of sensors including GPS, ultrasonic, and encoders for the wheels' angular motion detection. Safe navigation and interaction is implemented in the system. Collision avoidance information is preprogrammed into the embedded firmware. Also, this information can be overwritten by the robot's maintenance module inside the robot service centre. During operation, these sensors detect objects in their moving environment and examine obtained information with a collision avoidance database. If there is any matched information, the robot will first notify the user about the situation, enter a timeout period, and wait for further instruction from the doctor's side. If there is no instruction after the timeout, the robot will automatically modify its path to avoid collision. For continuous patient monitoring, two approaches have been adopted. The first approach is made possible by using a specially developed wireless measurement module. The transmitter module with a bio sensor is attached to the patient's body while the receiver unit is directly connected to the laptop via a USB port. The doctor can use the bio metering software module to activate the measurement service. This results in data transmission from the transmitter unit. When the data is available to the robotic system, the robot will first send the data directly to the bio metering software module on the doctor side. In addition, the robot also sends the same data set to the robot control centre for off-line checking and back-up purposes. Alternatively, the second measuring approach is accomplished using a custom 
made optical pulse oximetry sensor attached to the robot's end-effector. This type of sensor can be used to sense bio information such as haemoglobin, oxygen content and heart rate, etc. Using this approach, the doctor can control the position of the robot's end-effector, remotely, to obtain pertinent bio-data to determine the patient's health conditions including fever, infection, pneumonia and asthma, among others (King, 2003).

\subsection{Robot control centre}

The robot control centre is developed to enhance the level of interaction between the doctor, mobile robot, and system supervisor. For the current research goal, the robot control centre is responsible for the following sub-tasks:

- central database management system for patient data backup

- monitoring and book-keeping of mobile robot's health in real-time

- online web service for off-line patient data history

- preventive maintenance of robotic system

- firmware upgrading and embedded system maintenance.

The proposed robot control centre consists of a main PC server and three software modules depicted in Figure 4.

Figure 4 Component service for robot control centre (see online version for colours)

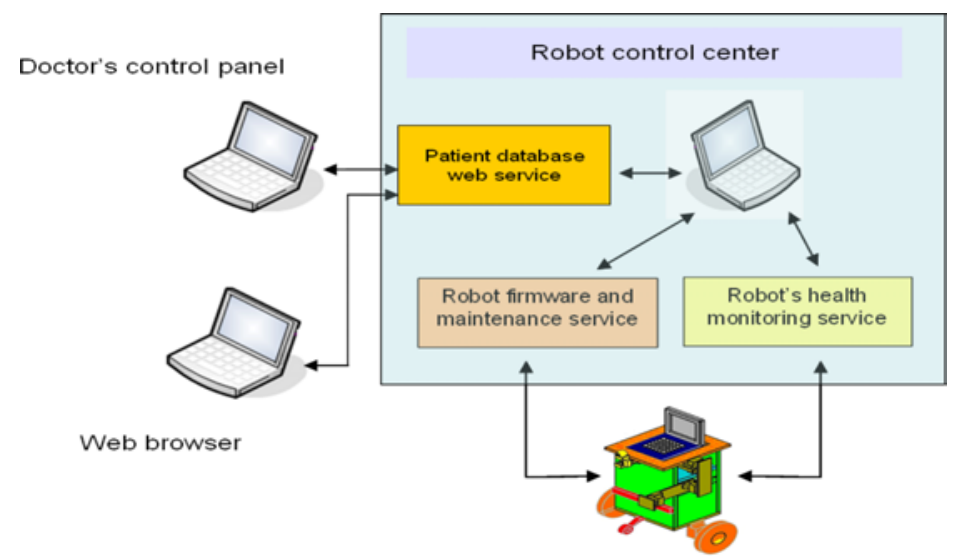

\section{System implementation}

\subsection{Robot's mechanical design}

The conceptual design and actual robot platform are shown in Figure 5. The proposed robotic platform has four main components. These include the robot's main body, the wheel base system, the robotic arm, and the modular embedded system housing, as shown in Figure 5. The body itself is made of eight pieces of Plexiglas structures. 
These parts are joined using L-shaped aluminium bars. The laptop, acting as the face of the robot, is placed on the top part of the structure. A two-degrees-of-freedom pan-tilt camera unit is connected to the top front of the body. Thus the video camera can be directly attached on the top of the pan-tilt unit. Detection of obstacles is accomplished by two types of sensors, ultrasonic and infrared. Ultrasonic sensors are attached to the middle front body to detect relative position of the obstruction. The infrared sensors are connected to the bottom part of the robot's body so that the robotic system is also able to detect obstacles that extend slightly above the ground.

Figure 5 Conceptual design and the actual mobile robot platform (see online version for colours)
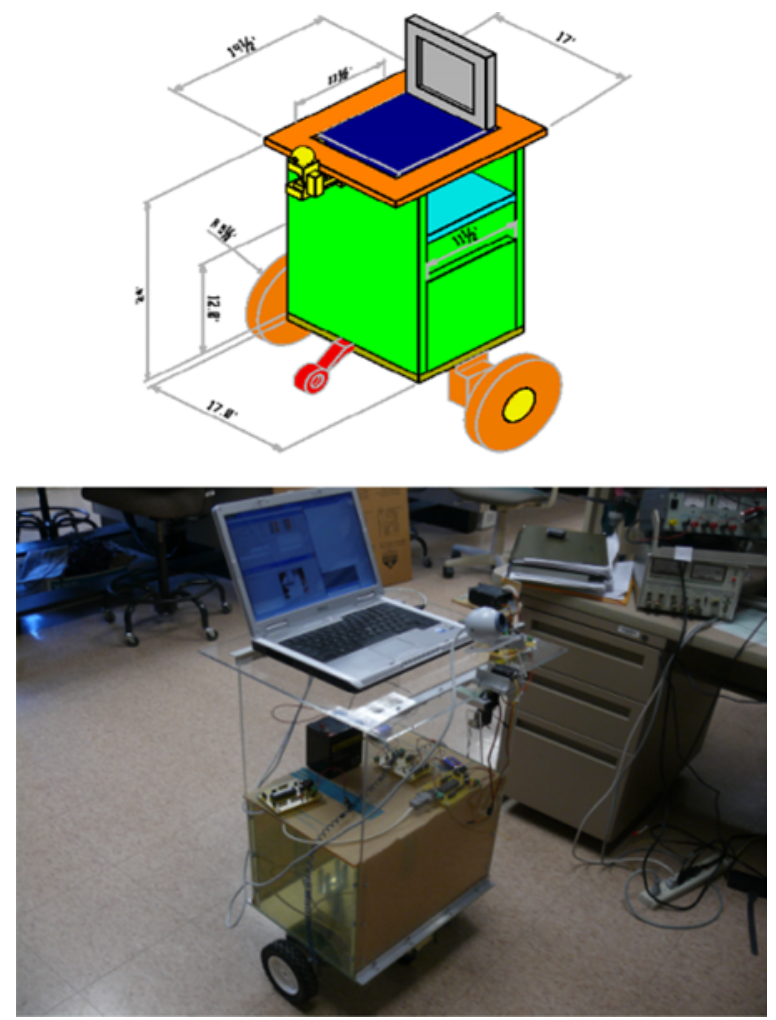

Two wheels with a differential drive control technique (He, 2005) are used for motion control of the wheel base system. The wheels are actuated by high torque DC motors with a gearbox system. Two omni-directional slack wheels are also connected to the back and front of the platform to improve the structural stability of the system. The robotic arm unit is also made from Plexiglas. The current configuration allows the doctor to virtually control the robot arm with four degrees of freedom. This includes three arm links rotations and an end-effector rotation. Each arm link is actuated by high torque SC-100 servo motors. From experimental observations, this level of torque is adequate for smooth arm movement in our prototype design. The structure also includes a storage space reserved for additional equipment and power unit. This storage part is located at the middle of the main body and can be used to house battery and multiple embedded systems. 


\subsection{Embedded hardware units for mobile robot}

Modularity and upgradeability are two factors for our design consideration. The proposed robotic system utilises a re-programmable embedded microcontroller with dedicated hardware components. Firmware revision can be easily upgraded for each controller unit via the robot service centre. Moreover, each controller unit can be reconfigured to perform separate automatic operations, independently. These include motion control, wireless data communication, sensing and actuation. The architecture of the embedded electronics of the multi-controller modules for the robotic assistant is shown in Figure 6. Each sub-unit has a dedicated microprocessor and directly communicates with the laptop via USB port connector. For communication between the controller and the laptop, a USB-to-serial communication adaptor is employed. This interfacing technique eliminates the need to write low-level intensive code for USB communication. Thus, the communication can be established as if there were two serial transmissions at both sides. Moreover, serial communication firmware code is reliable and easy for software debugging.

Figure 6 Embedded architecture of mobile robot (see online version for colours)

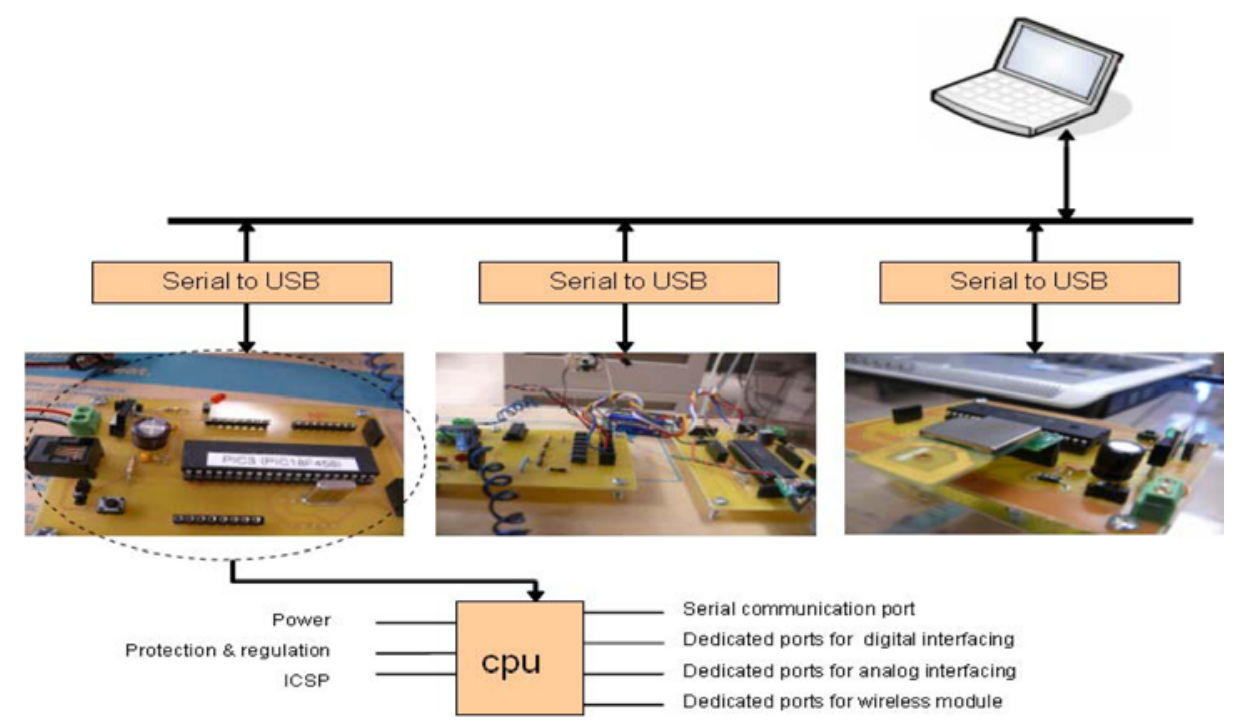

\subsubsection{Generic controller unit}

Each generic controller unit has its own microprocessor and peripheral interfacing connector. In particular, a low power PIC18F458 microcontroller from MICROCHIP is selected as the main CPU. The chip features stable 10 MIPS processing speed, 33 digital I/O pins, 32 Kbytes flash program memory and 256 byte of EEPROM for persistent data stream. It has several peripheral modules which can be reconfigured to support sensing and actuation tasks. The current embedded controller configuration also contains an ICSP programming circuit. Thus, remote firmware upgrading and debugging from the main robot control centre can be made online. Figure 7 illustrates the interfacing technique and reconfigurable scheme among several hardware components of the robotic system. 
Tele-doc: an open architecture robotic system for telemedicine applications 397

Figure 7 Embedded system interfacing technique (see online version for colours)

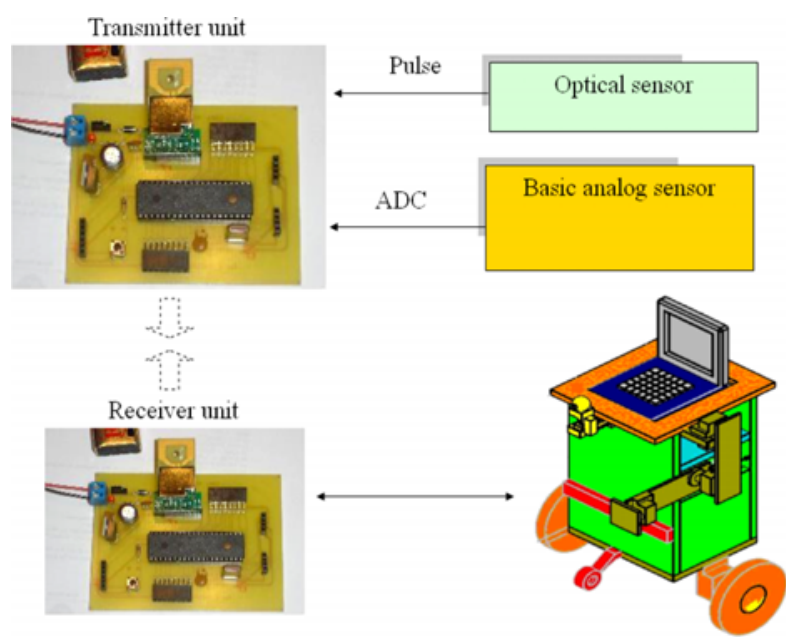

\subsubsection{Wireless data acquisition unit}

In addition to the bio-sensor at the robot arm's end-effector, a wireless transceiver is provided that offers an effective way for wireless continuous data monitoring, as shown in Figure 8. This hardware module consists of two units that can be directly connected to bio-sensor devices and their embedded controller on the robot platform. More specifically, one wireless controller unit is configured as the transmitter which directly connects to bio-sensors for data acquisition. The other wireless controller unit is configured as the receiver and directly connects to the laptop notebook via a USB port.

Figure 8 The embedded prototype for wireless data acquisition (see online version for colours)

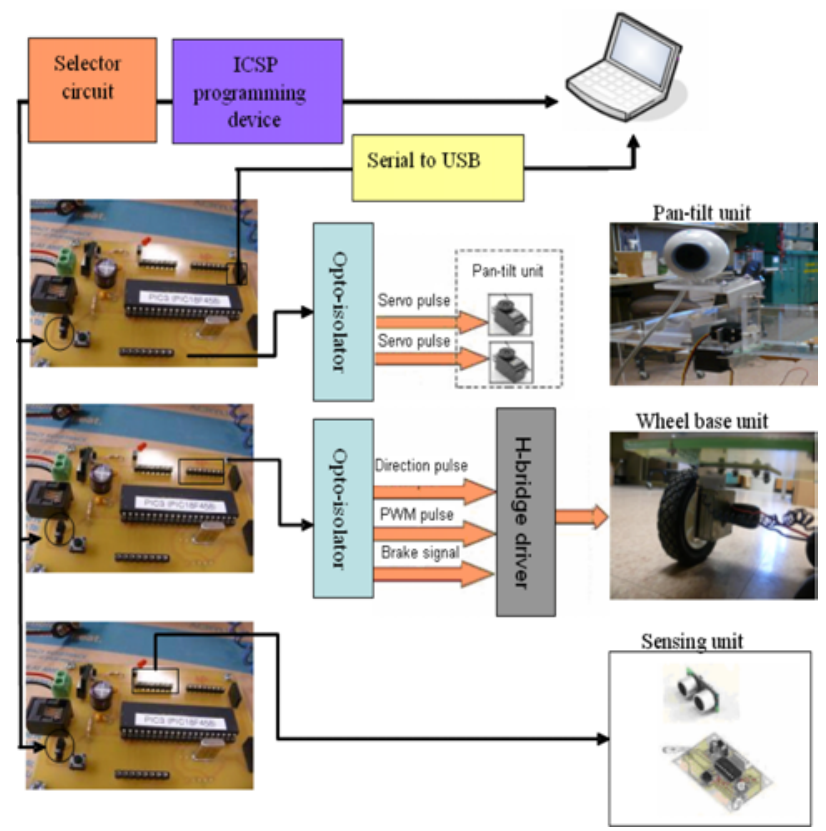


Each wireless controller unit consists of a generic controller and a wireless transceiver module. The transceiver module is TRW-24G which provides communication throughput up to $1 \mathrm{Mega}-\mathrm{bit} / \mathrm{second}$. However, our experiments showed robust communication speed with $256 \mathrm{Kilo-bit/s} \mathrm{setup.} \mathrm{The} \mathrm{unit} \mathrm{can} \mathrm{be} \mathrm{programmed} \mathrm{to} \mathrm{work} \mathrm{simultaneously} \mathrm{as} \mathrm{both}$ transmitter and receiver. It can also be used to connect to multiple sensor devices to provide up to eight channel measurements. Doctors can use a bio metering software module to obtain wireless data from the mobile robot. Upon receiving the command from the mobile robot, the raw data will be transmitted to the receiver at the robot side. The data will directly go to the bio metering software. The same set of data will also be transmitted to the robot control centre for backup. The communication protocol for the transmitter and receiver units is shown in Figure 9.

Figure 9 Transmission protocol for wireless measurement unit (see online version for colours)

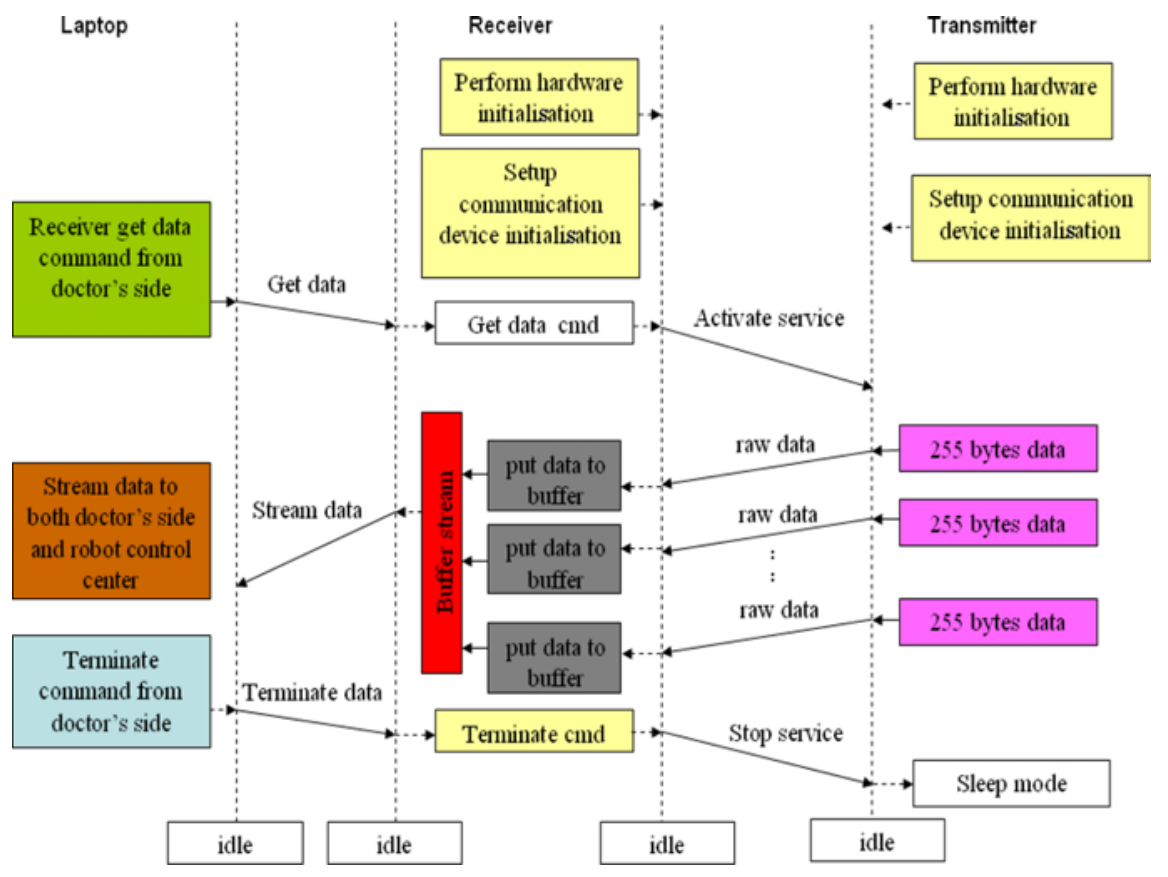

\subsubsection{Doctor-robot interaction unit}

In addition to the GUI's command button in the doctor's control panel, a Wii remote joystick has been used to provide a higher level of flexibility for the doctor- robot interaction, as in Figure 10.

A set of developed device libraries is provided to detect the stream of wireless data coming from the joystick device, as shown in Figure 11. For the current implementation, information such as button pressing for acceleration is utilised for the system. Also, the doctor's hand movements can be wirelessly recorded. This information can then be translated into a motion command usable by a webcam and the robot's arm control during a real-time visit. The communication between the Wii remote and the $\mathrm{PC}$ is made possible by a Bluetooth to USB conversion device (IT-Zone, 2008). Details of the device driver structure and data format used by the remote control can be found in Wiili (2008). 
Figure 10 Doctor-robot interaction unit (see online version for colours)
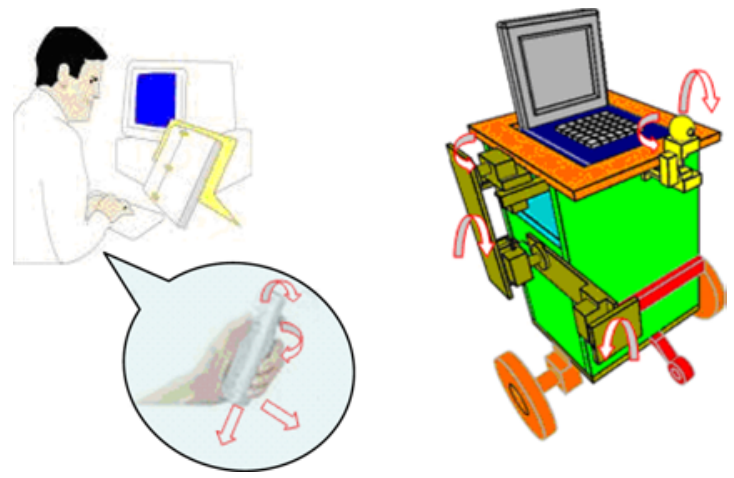

Figure 11 Rapid prototyping software architecture (see online version for colours)

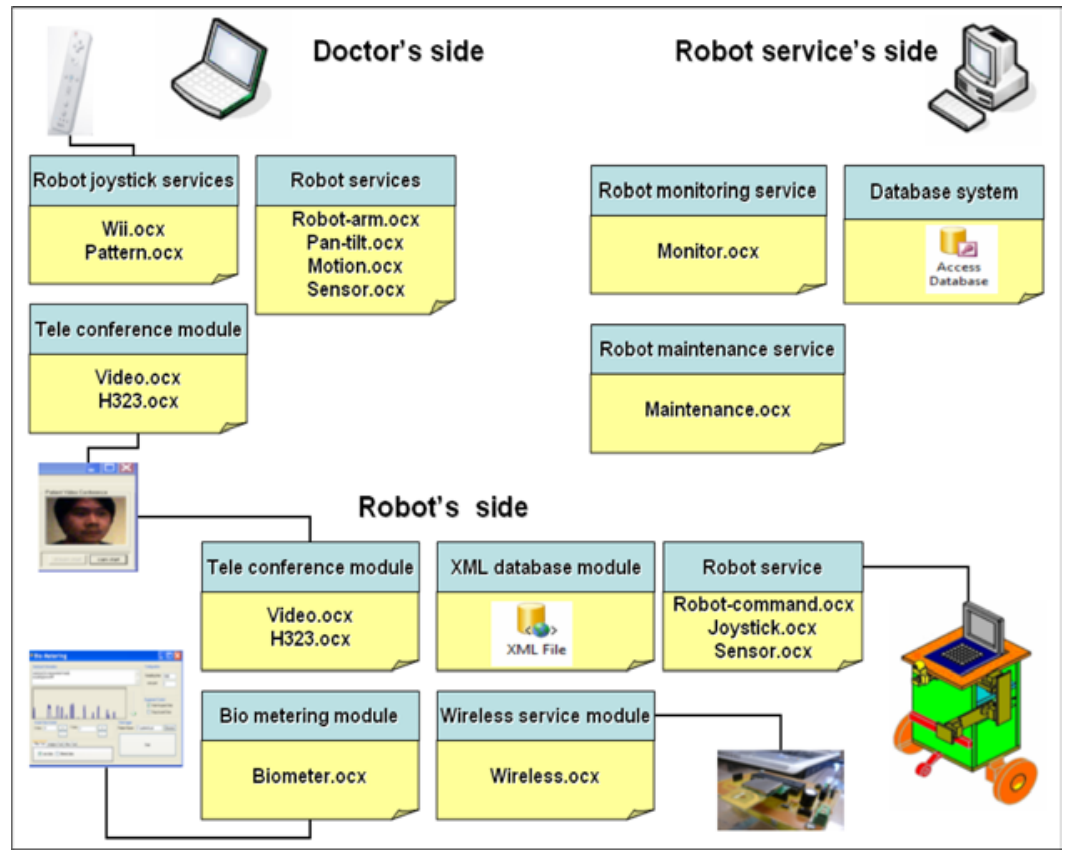

\subsubsection{Power consideration}

There are three separate power units for motion control, embedded systems, and the laptop notebook, respectively. Power to drive the DC motor and servo motors is derived by one main SLC battery type with $12 \mathrm{~V} / 5 \mathrm{Ah}$. This battery weighs about two pounds while the second SLA battery with the same power capability serves as the main power source for all embedded systems. The third battery supports the power source for the laptop. For the current prototype, we implement a power protection scheme by adopting a power switch breaker set for each individual subsystem. If the breaker trips, the monitoring circuit with the built-in battery will notify the main computer laptop. For current configuration, the power monitoring software module will log this event and notify the robot control centre about the situation. 


\subsection{Software framework}

The proposed software application is based on the multi-tier client server. There are three tiers including a doctor tier, a mobile robot tier, and a robot control centre tier for the proposed development domain. The communication protocols are separately developed for each system. This includes a tele-robot platform control, a robotic health report, a voice and video communication, a patient's wireless data service, and a robot control centre service. In the current stage of development, all software modules are developed mainly using Microsoft visual basic 6.0 and visual c\# 2005. This development environment requires both run-time development library (Visual Basic, 2008), and dotnet 2.0 framework (DOTNET, 2008) installation. For any time-critical and low-level $\mathrm{I} / \mathrm{O}$ task, visual $\mathrm{c}++$ is employed to create a dynamic software library for low-level hardware access. All of the software components are packaged as activeX control (ocx) (Surfthenetsafely, 2008) that serves each sub-task.

\subsubsection{Tele-robot control service}

A high-level communication layer usually provides more flexibility for software development. However, a low-level communication protocol such as TCP/IP allows the developer to embed more information and it usually works faster. The software protocol for remote access has been developed using winsock control (Sockets, 2008) provided in Microsoft visual studio suite. The low-level communication protocol is designed based on the end-point communication. At the high level, a command interpreter and a string-based command protocol are provided, as shown in Figure 12.

Figure 12 Robot's string-based communication protocol (see online version for colours)

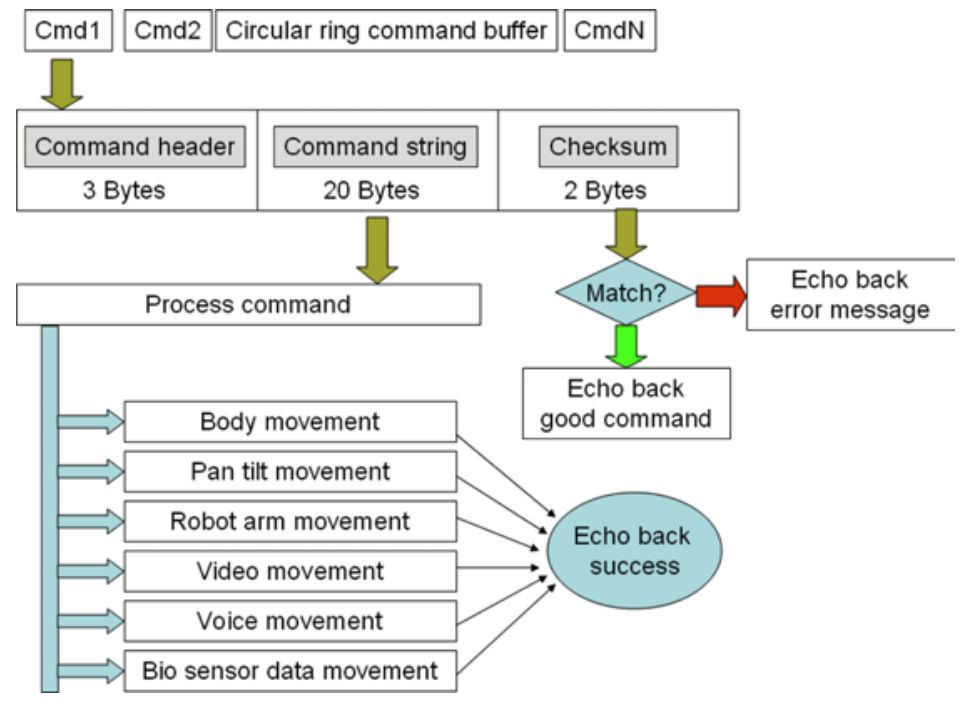

\subsubsection{Teleconference service}

This software module is developed for point-to-point video and voice communication between the robot side and the doctor side, respectively. Image capturing from both sides are pre-configured for 15 frames/s. Raw images are encoded by open standard video 
codec h263+ (Keith, 2004). Voice communication is developed based on the open standard h323 technology (OpenH323, 2008). The doctor can call the robot as if they were on a land line by using a login account. Moreover, this software module is packaged as activeX control which can be easily deployed for future image processing applications, including patient's face-emotion detection and colour-based disease diagnosis, etc.

\subsubsection{Patient data structure}

The data structure of human bio information is developed based on the XML concept. Since the bio data is hierarchical by nature, the XML data format allows us to embed the information in a hierarchical form. In addition, the XML data format can be easily exchanged within the network environment. Figure 13 illustrates an example of patient's bio data in XML format.

Figure 13 Patient XML data structure

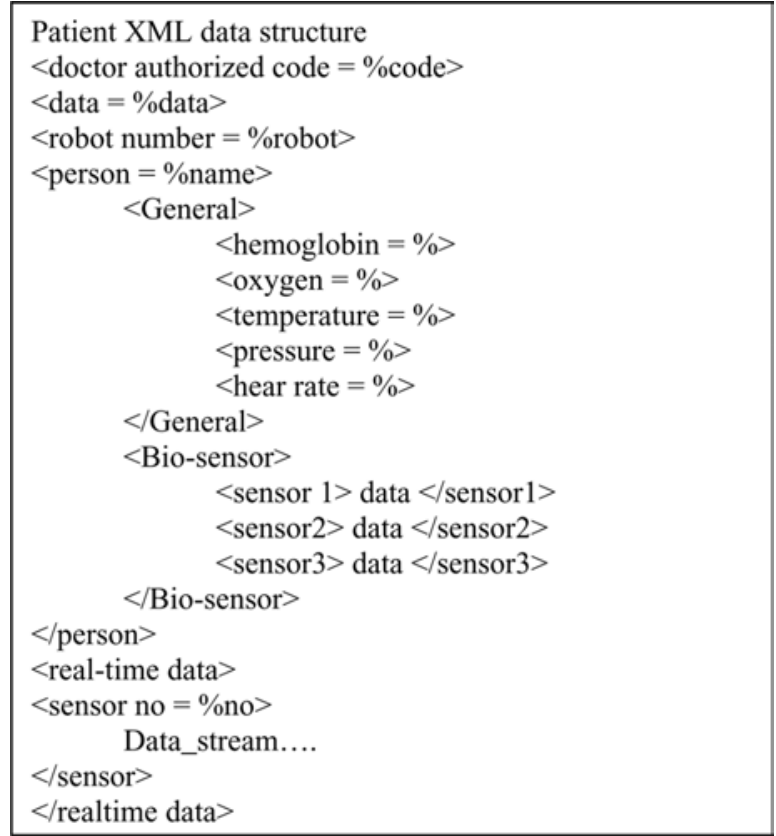

When the doctor requests the patient's raw bio data from the mobile robot system, the raw data from the wireless measurement is first stored into the patient database system inside the robot platform. Then, the raw data will be sent to both the doctor tier and the robot service centre. The data going to the robot service centre is in the XML data format and is later converted and recorded into the Microsoft Access database system. Data conversion is made possible by using ADO.net technology (ADONET, 2008). One software adaptor module is then implemented to convert the XML database format into the normal RDBMS. The same raw data set is then transmitted to the doctor side and is presented using the bio-metering software module. 


\subsubsection{Robot service centre}

We adopted C\#.NET framework as the core internet framework for our proposed robot system service centre. In addition to our client and server software framework, this software module extends the capability of the system by allowing the doctor and the health practitioner to have access to the mobile robot information from the robot service centre. This implementation requires an ASP.net and our developed classes of object with embedded web-service routines. The doctor can use the web browser program to have access to both the patient's current condition and the off-line bio-data. For the current implementation, the database engine is implemented on top of the Microsoft Access engine. All three service modules, including the patient's database system, the robot's health monitoring system, and the firmware debugging service, are written in $\mathrm{c \#}$ managed code. Clearly, this data information can be easily integrated into the hospital's care information.

\section{Non-invasive biomedical sensors}

Bio information such as body temperature, systolic-diastolic blood pressure, cardiac and respiratory rhythms, and oximetry are necessary for determining the patient's health condition. There are many essential bio-data measurements that require invasive tools. A robotic assistant for tele-medicine applications must be equipped with non-invasive bio-sensors. Over the past years, much effort has been placed on developing non-invasive measurement techniques for biomedical applications (King, 2003; He, 2005; Bachiochi, 2005; Jubran, 1999; Webster, 1997; Puentes et al., 2007; Korsunsky et al., 2005; Thrush et al., 2004; Olesberg, 2004; Macknet et al., 2007; Kraitl and Ewald, 2005; Ewald and Kraitl, 2007; Spigulis et al., 2004; Spigulis, 2005; March, 2001; Waynant and Chenault, 1998; Cho et al., 2004; Yamakoshi et al., 2007). A number of such devices are based on utilising optical techniques. Unlike invasive methods, the optical characteristic of a sensing device makes it possible for a doctor to remotely monitor the patient's bio data. However, the sensor itself must be reliable and robust for measurement purposes. Moreover, any error due to measurement in the presence of noise or disturbance might deteriorate the quality of the measurement information.

Here, a number of non-invasive bio-measurement tools were considered for tele-medicine applications. One such sensor was the pulse oximetry sensor to determine the oxygen level in the patient's blood. The sensor was experimentally tested in the laboratory for use with the proposed tele-robotic system. A linear filtering mechanism was considered for de-noising the measured signals in order to obtain accurate measurements. Based on results in Bachiochi (2005) and Jubran (1999), an oximetry sensor can be constructed with any light-to-frequency converter device. To measure oxygen level and heart rate, a TSL257 sensor from TAOS was selected to create a reliable oximetry sensor unit. The principle of operation of this optical sensor is as follows. A pair of red and infrared light emitters is used to beam light through a subject's finger. The sensors in the TSL257 light-detector sense the two lights beneath the finger and generate proportional voltages. Oxygen level can then be calculated using the relationship between the blood's oxygen level and the absorption level of the red and infrared light detectors. Equations governing this relationship are given in Webster (1997). The developed sensor can either be directly attached to the robotic end-effector or 
connected to a wireless controller unit attached to the patient's body. The data sampling period for the tested device was configured at $100 \mathrm{~Hz}$ for normal measurement operation. Additional filtering and calibration are required for accurate measurement of the blood's oxygen level from the measured data (Bachiochi, 2005; Jubran, 1999; Webster, 1997) and for its incorporation into the robotic system.

There are various optical sensing methods for continuous non-invasive measurement of other bio-data, such as haemoglobin, glucose and many other blood contents (Puentes et al., 2007; Korsunsky et al., 2005; Thrush et al., 2004; Olesberg, 2004; Macknet et al., 2007; Kraitl and Ewald, 2005; Ewald and Kraitl, 2007; Spigulis et al., 2004; Spigulis, 2005; March, 2001; Waynant and Chenault, 1998; Cho et al., 2004; Yamakoshi et al., 2007; Jaundice Meter, 2008; Yasuda et al., 2003; Singh and Chauhan, 1989). Non-invasive haemoglobin measurements have many potential advantages including the accurate and continuous monitoring of anaemia. In Macknet et al. (2007), Kraitl and Ewald (2005) and Ewald and Kraitl (2007), multiple-wavelengths were used for the measurement of various components in the blood. Alternatively, in Spigulis et al. (2004) and Spigulis (2005) a multiple-channel strategy utilising measurement at different body locations, such as fingertips, earlobes, forehead, and forearms, was used to monitor cardiovascular conditions. From the above observations (Kraitl and Ewald, 2005; Ewald and Kraitl, 2007; Spigulis et al., 2004; Spigulis, 2005), one can speculate that, given enough computational power, a multi-channel, multi-wavelength bio-measurement strategy could provide a much more reliable monitoring of the cardiovascular system than each method alone.

Measurement of glucose level in the blood is a necessary test for people with diabetes, which typically requires drawing blood several times a day by pricking the fingertips. In order to avoid such an inconvenient process, various non-invasive techniques have been considered for the measurement of the glucose concentration in the blood (March, 2001; Waynant and Chenault, 1998; Cho et al., 2004; Yamakoshi et al., 2007). However, these methods require extensive clinical testing and none of them are ready for commercialisation yet. It was noted, though, that such techniques will soon be improved and will be commercially available for medical applications (Waynant and Chenault, 1998).

Other types of non-invasive bio-sensing techniques were also considered in the literature (Jaundice Meter, 2008; Yasuda et al., 2003; Singh and Chauhan, 1989; Bruno and Scalart, 2005). For example, in Jaundice Meter (2008), a new optical measurement device was commercially introduced for detection of jaundice in newborn babies. In Singh and Chauhan (1989), a non-invasive ultrasonic method was proposed for continuous measurement of body temperature in clinical applications. Also, in Bruno and Scalart (2005), an amplitude and frequency (AMFM) demodulation technique was developed for estimation of cardiac and respiratory rhythms. Clearly, there are many techniques available for the development of non-invasive bio-sensors. Our future plans include the development and testing of a number of optical bio-sensors that can be implemented in our prototyped robotic system for tele-medicine applications.

\section{Conclusion}

We proposed and implemented an open architecture mobile robotic system for tele-visit, and remote health monitoring and diagnosis. The system includes reconfigurable 
real-time software and embedded processing and internet-based wireless communication modules. The robot's control service centre is integrated into a working environment to provide off-line patient information and to perform preventive maintenance of the robotic platform. The current working prototype has been tested in a laboratory environment. Our experimental results showed that the automatic and manual control of robot platform can be easily performed remotely by a human operator. Hence, the proposed tele-robotic system can be employed as an assistant tool for doctors to provide an alternative level of mobile health monitoring and diagnosis. The open architecture was considered for modular software development and low cost reprogrammable embedded systems for robotic platform. Also, an alternative method for the doctor and robot interfacing was developed. The method used a Bluetooth special joystick controller to provide a natural and more comfortable interaction with the tele-robot for healthcare applications in hospitals and clinical environments. Examples of several possible bio-sensors that could be incorporated in the proposed tele-robotic system were also discussed.

\section{References}

ADONET (2008) ADO.NET 1.1/2.0, Microsoft MSDN Data Platform Development Center, Accessed May 2008, http://msdn2.microsoft.com/en-us/data/aa937722.aspx

Bachiochi, J. (2005) 'Light-to-frequency conversion pulse and oxygen content', Circuit Cellar Magazine, No. 174, January, pp.68-71, http://www.circuitcellar.com/library/print/0105/ Bachiochi174/index.htm

Bruno, S. and Scalart, P. (2005) 'Estimation of cardiac and respiratory rhythms based on an amfm demodulation and an adaptive eigenvector decomposition', The 13th European Signal Processing Conference (EUSIPCO), Antalya, Turkey, September, Paper \# cr1416.

Cho, O.K., Kim, Y.O., Mitsumaki, H. and Kuwa, K. (2004) 'Noninvasive measurement of glucose by metabolic heat conformation method', Clinical Chemistry, Vol. 50, August, pp.1894-1898.

Coulston, C. (2005) 'Electronic angle measurement', Circuit Cellar Magazine, No. 179, June, pp.14-18, http://www.circuitcellar.com/magazine/179toc.htm

DOTNET (2008).NET Framework Microsoft MSDN .NET Framework Developer Center, Accessed May 2008, http://msdn2.microsoft.com/en-us/netframework

Ewald, H. and Kraitl, J. (2007) 'Optical sensor technology for a noninvasive medical blood diagnosis', in Coté, G.L. and Priezzhev, A.V. (Eds.): Proceedings of the SPIE, Optical Diagnostics and Sensing VII, Vol. 6445, January, paper \# [6445-10], San Jose, CA.

He, S. (2005) 'Feedback control design of differential-drive wheeled mobile robots', Proceedings of the 12th International Conference on Advanced Robotics (ICAR '05), July, Seattle, WA, pp.135-140.

IT-Zone (2008) Bluetooth Technology, IT-Zone Online Publication, Accessed May 2008, http://it-zone.ifastnet.com/Articles/Communications/BlueTooth.html

Jaundice Meter (2008) Jaundice Meter - JM103, Konica-Minolta Online Brochure, Accessed May 2008, www.konicaminolta.com/instruments/download/catalog/medical/pdf/jm103_e7.pdf

Jubran, A. (1999) 'Pulse Oximetry', Critical Care, Vol. 3, No. 2, May, pp.R11-R17, Published Online, http://www.pubmedcentral.nih.gov/articlerender.fcgi?artid=137227

Keith, J. (2004) Video Demystified - A Handbook for the Digital Engineer, 4th ed., Elsevier, New York, NY.

King, R. (2003) 'Optoelectronic sensors in medical applications', Sensor Magazine, Vol. 20, No. 9, September, Published Online: http://archives.sensorsmag.com/articles/0903/32/ 
Tele-doc: an open architecture robotic system for telemedicine applications 405

Korsunsky, V., Romanov, V., Starodub, M. and Voytovych, I. (2005) 'Automated optoelectronic sensors for medicine and biology', IEEE Workshop on Intelligent Data Acquisition and Advanced Computing Systems: Technology and Applications (IDAACS), September, pp.413-416.

Kraitl, J. and Ewald, H. (2005) 'Optical non-invasive methods for characterization of the human health status', 1st International Conference on Sensing Technology, Palmerston North, New Zealand, 21-23 November, pp.466-470.

Macknet, M.R., Norton, S., Kimball-Jones, P., Applegate II, R., Martin, R. and Allard, M. (2007) 'Continuous non-invasive measurement of hemoglobin via pulse co-oximetry', Annual Meeting of the Society for Technology in Anesthesia (STA), January, Online Archive, $\mathrm{http}: / /$ www.anestech.org/publications_abtracts.htm\#STA2007

March, W.F. (2001) 'A noninvasive ocular glucose sensor', Diabetes Technology and Therapeutics, Vol. 3, No. 2, June, pp.209-210.

Melek, W.W., Sadeghian, A., Najjaran, H. and Hoorfar, M. (2005) 'A neurofuzzy-based expert system for disease diagnosis', IEEE International Conference on Systems, Man and Cybernetics, Vol. 4, October, pp.3736-3741.

Moret-Bonillo, V., Mosqueira-Rey, E. and Alonso-Betanzos, A. (1997) 'Information analysis and validation of intelligent monitoring systems in intensive care units', IEEE Transaction on Information Technology in Biomedicine, Vol. 1, No. 2, June, pp.87-99.

Murray, R.M., Li, Z. and Sastry, S.S. (1994) A Mathematical Introduction to Robotic Manipulation, CRC Press, Boca Raton, Florida.

Olesberg, J.T. (2004) 'Applications of long-wavelength sources and detectors for medical monitoring', Proceedings of Electrochemical Society, State of the Art Program on Compound Semiconductors (SOTAPOCS), Vol. 2, 12 May, pp.210-217.

OpenH323 (2008) Welcome to the OpenH323 Project, OpenH323 Online Publication, Accessed May, http://sourceforge.net/projects/openh323

Puentes, J., Bali, R.K., Wickramasinghe, N. and Naguib, R.N.G. (2007) 'Telemedicine trends and challenges: a technology management perspective', Int. J. Biomedical Engineering and Technology, Vol. 1, No. 1, pp.59-72.

Sagiroglu, S., Yilmaz, N. and Wani, M.A. (2006) 'Web robot learning powered by bluetooth communication system', 5th International Conference on Machine Learning and Applications (ICMLA'06), December, Orlando, FL, pp.149-156.

Singh, V.R. and Chauhan, S. (1989) 'An ultrasonic non-invasive temperature monitor', Proceedings of the Annual International Conference of the IEEE Engineering in Medicine and Biology Society, Images of the Twenty-First Century, Vol. 5, November, pp. 1628-1629.

Smith, C.D. and Skandalakis, J.E. (2005) 'Remote presence proctoring by using a wireless remote-control videoconferencing system', Surgical Innovation, Vol. 12, No. 2, June, pp.139-143.

Sockets (2008) WinSock Development Information, Sockets Online Publication, Accessed May, http://www.sockets.com/

Spigulis, J. (2005) 'Optical noninvasive monitoring of skin blood pulsations', Applied Optics, Vol. 44, No. 10, April, pp.1850-1857.

Spigulis, J., Erts, R. and Ozols, M. (2004) 'Optical multi-channel monitoring of skin blood pulsations for cardiovascular assessment', in Cohn, G.E., Grundfest, W.S., Benaron, D.A. and Vo-Dinh, T. (Eds.): Proceedings of SPIE, Advanced Biomedical and Clinical Diagnostic Systems II, Vol. 5318, July, pp.133-139.

Surfthenetsafely (2008) ActiveX in Windows - What is it?, Surf The Net Safely Online Publication, Accessed May, http://surfthenetsafely.com/activex.htm

Thacker, P.D. (2005) 'Physician-robot makes the rounds', Journal of the American Medical Association, Vol. 293, No. 2, January, p.150. 
Thrush, E., Levi, O., Cook, L.J., Smith S.J. and Harris Jr., J.S. (2004) 'Greater than $10^{6}$ optical isolation in integrated optoelectronic fluorescence sensor', 26th Annual International Conference of the IEEE Engineering in Medicine and Biology Society (IEMBS), San Francisco, CA, Vol. 1, 1-5 September, pp.2080-2081.

Trahanias, P., Burgard, W., Argyros, A., Hahnel, D., Baltzakis, H., Pfaff, P. and Stachniss, C. (2005) 'Web-operated mobile robots for tele-presence in populated exhibitions', IEEE Robotics and Automation Magazine, Vol. 12, No. 2, June, pp.77-89.

Vannoni, M. and Straulino, S. (2007) 'Low-cost accelerometers for physics experiments', Eur. J. Phys., Vol. 28, No. 5, September, pp.781-787.

Visual Basic (2008) Visual Basic Runtime Files, Microsoft Help and Support, Accessed May, http://support.microsoft.com/gp/vbruntime/en-us

Waynant, R.W. and Chenault, V.M. (1998) 'Overview of non-invasive fluid glucose measurement using optical techniques to maintain glucose control in diabetes mellitus', IEEE Lasers and Electro-Optics Society Newsletter, Vol. 12, No. 2, April, http://www.ieee.org/organizations/ pubs/newsletters/leos/apr98/overview.htm

Webster, J.G. (1997) Design of Pulse Oximeters, Institute of Physics (IOP) Publishing, Bristol.

Wiili (2008) Wiimote, Wiili Online Publication, Accessed May, http://www.wiili.org/index. $\mathrm{php} /$ Wiimote

Yamakoshi, Y., Ogawa, M., Yamakoshi, T., Satoh, M., Nogawa, M., Tanaka, S., Tamura, T., Rolfe, P. and Yamakoshi, K. (2007) 'A new non-invasive method for measuring blood glucose using instantaneous differential near infrared spectrophotometry', 29th Annual International Conference of the IEEE Engineering in Medicine and Biology Society (EMBS), 22-26 August, pp 2964-2967.

Yasuda, S., Itoh, S., Isobe, K., Yonetani, M., Nakamura, H., Nakamura, M., Yamauchi, Y. and Yamanishi, A. (2003) 'New transcutaneous jaundice device with two optical paths', Journal of Perinatal Medicine, Vol. 31, No. 1, January, pp.81-88. 